\title{
Complexity and aesthetic pleasure in websites: an eye tracking study
}

CHASSY, P. Psychology, University of Liverpool

FITZPATRICK, J. V. Department of Psychology, Liverpool Hope University

JONES, J. A. Department of Psychology, Liverpool Hope University

PENNINGTON, G., Department of Psychology, Liverpool Hope University

The internet is an ever-expanding medium of communication; capable of connecting users with masses of information and countless services. In such a popular and competitive market, it is imperative that websites are both practical and pleasant to navigate. Using an eye-tracker, we obtained objective measurements of complexity with subjective ratings of complexity and aesthetics, to explore judgments arising from the perception of university homepages. As expected, high levels of perceived complexity resulted in low aesthetic ratings. Interestingly, fixation count correlated negatively with complexity and positively with aesthetics; a novel finding. We argue that high complexity and negative aesthetic appraisals are a result of cognitive overload due to the limited capacity of working memory. We suggest ways in which web designers can avoid information overload, by complying with the known limits of human cognition, to maximize the positive interaction between webpage and user.

Interaction Science Key Words: human-computer interactions, behavioral, eye-tracker, linear regressions, websites, prototypicality, visual complexity, aesthetics.

DOI: 10.24982/jois.1713017.003

\section{INTRODUCTION}

Research within Human-Computer Interaction (HCI) aims to understand and improve the interactive experience of users with various aspects of computer technology, such as software, smart phone interfaces, and websites. Regarding the latter, the internet is a medium of communication, connecting users with information in the form of products, learning materials, and services. With such an integral medium, it is essential that designers strive to create websites that are both functional and pleasant to navigate. To be functional, the website should display all necessary information. Yet, too much information may result in high visual complexity which impacts the quality of perception, ultimately disengaging users. In the present study, we sought to shed further light on the relationship between visual complexity and aesthetics, which in past research, has produced contradictory findings $[4,19,28,39]$.

Complexity is a measure of the number of items and potential number of interactions within a system. Complexity is thus an intrinsic characteristic of a given system or object. The brain, for example, is a typical highly complex system for its behavior is dictated by numerous components that interact with different dynamics [34]. The definition of complexity can be extended to living systems in general [33] and to many purely mechanical systems. In light of this definition, websites are made of numerous elements that interact semantically or spatially. To this level of complexity, we can add factors such as font size, the number of images, and, key to visual research, the spatial distribution of all items. All these parameters contribute to make the website a complex object [6]. The viewer has to explore the website visually to make sense of its content. At a psychological level, the cognitive system deals with complexity by breaking down the visual scene into meaningful, 
recognizable components. Pioneering research in the field of chess expertise has been carried out by Chase and Simon [7] and now constitutes a major strand in accounting for acquisition of high level performance in humans [8]. In this context, perceptual complexity refers to the subjective estimate of complexity that emerges from the interaction between the actual complexity of the object and the ability of the perceiver to visually structure the scene into meaningful elements [5]. Perceptual complexity in websites has already been acknowledged by the HCI community $[12,27]$. That complexity influences perception has been documented in a variety of studies $[4,8,18]$. Recently, complexity has invited ample study in website perception specifically $[13,15,24,25,39$, 41, 42]. Complexity has been shown to influence aspects such as effectiveness [13], preference, and ease of navigation of websites [24]. Visual complexity is an essential aspect of website design, as the degree to which a website is complex impacts its usability [36], which in turn may affect customer satisfaction [2] and credibility [20]. Multiple objective factors including percentage of white space [15] and number of links and home page length [14] contribute to website complexity. In parallel, research shows that subjective complexity influences several factors such as attention, users' attitudes towards a company, and intent to purchase from home pages [13]. Evidence suggests that reducing complexity in the form of implementing easily identifiable icons on webpages can lead to greater usability [10]. Such studies illustrate the centrality of complexity in websites. Thus, striving towards a greater understanding of its perception is a useful endeavor for informed, effective web design.

While the usability of websites and various interfaces is a strong focus of studies concerned with $\mathrm{HCI}$, website aesthetics are likewise an important consideration for design. Aesthetic is an emotion derived from the appreciation of visual features of a given stimulus. It is a subjective measure of a viewer's pleasure. Aesthetic pleasure is commonly measured by asking participants to rate on a Likert scale the aesthetic features of visual items [38]. The general relationship between complexity and aesthetic pleasure is not new in the literature and has benefitted from investigation using a varied range of stimuli $[3,4,17]$. Early research on neutral structures such as polygons reports a relationship in the form of an inverted u-curve, where the peak of aesthetic pleasure is matched with a moderate degree of complexity [3]. The inverted u-curve has also been documented in complexity of art $[9,19,41]$. Specifically in web design, aesthetic features have been shown to play a key role $[11,22,43,44,46]$. As such, an awareness of the importance of aesthetics can be seen increasingly throughout the HCI research. Some studies report an inverted u-curve relationship between complexity and aesthetics [13, 24, 41]. A study by Geissler, Zinkhan, and Watson [13] manipulated homepage screens, links, and graphics, and found that preference was greatest for websites falling within a moderate level of complexity. In addition, Wang [41] likewise reported an overall preference for moderate complexity for children; although the introduction of gender as a control resulted in some preference differences between genders. However, additional research on website complexity challenges the classic relationship theorized by Berlyne [4], as studies on websites have often yielded a negative linear relationship between complexity and aesthetic pleasure $[25,28,39$, 40]. That is, the optimum level of aesthetic pleasure corresponds with the lowest degree of perceived complexity. For instance, Pandir and Knight [28], finding no support for Berlyne's theory of aesthetics, discovered a significant negative correlation between pleasure and complexity elicited from the perception of websites. Tuch, Bargas-Avilaa, Opwis, and Wilhelm [39] further uncovered a negative relationship between complexity and affective valence, and in addition, found that recognition performance is best with websites of low complexity. A study by Stevenson, Bruner, and Kumar [35] investigated the effects of webpage backgrounds on the effectiveness of goods advertisement, and showed that simple backgrounds impact more positively than complex backgrounds on aspects such as attitudes towards the website and purchase intention. Tuch, Presslaber, Stöcklin, Opwis, and Bargas-Avila [40] suggest that the typical negative relationship between complexity and aesthetics with websites may be owing to the fact that websites are 
generally more complex than the stimuli used in Berlyne's research, creating a restriction of range in complexity, with websites falling within the right side of the inverted u-curve. Taken together, the findings suggest that the relationship between aesthetics and complexity is dependent upon the type of stimuli used. In websites, it appears that simplicity is largely favored over complexity.

Although much previous research has used subjective measures involving the rating of stimuli [31], eye tracker studies are becoming increasingly common in HCI, e.g. [41]. Eye tracking enables the researcher to objectively map the sequence of gaze-shifting, as well as measuring where an individual's attention falls [29]. Obtaining objective measures of the number of eye fixations used to extract visual information may be indicative of the stimulus' level of complexity. For instance, Wang, Yang, Liu, Cao, and Ma [42], have reported a correlation between stimulus complexity and fixation count, through findings that when conducting simple tasks on websites, an increase in complexity leads to more fixations. The positive relationship between stimulus complexity and fixation count has likewise been demonstrated using images of cars as stimuli [9]. Implied through the results of the studies is that the more complex a stimulus, the more fixations required to process the information. Conversely, stimuli that are perceived as simple should require less fixations in order to extract the relevant information. In light of this, presently, it was expected that objectively measured complexity as number of eye fixations (fixation count) would correlate with subjective ratings of complexity, where for instance, websites with high ratings were expected to invite more eye fixations. Complexity was also predicted to be related to aesthetics, with the expected function of a negative linear form. In line with this, it seemed reasonable that the most positively received sites, being the least visually complex, would invite fewer fixations than sites of higher complexity.

\section{EXPERIMENTAL AND COMPUTATIONAL DETAILS}

\subsection{Participants \& Task}

A total of 40 participants were included in this study. Ages ranged from 18 to 41 years $(M=25.18$ years old, $\mathrm{SD}=6.98$ years old), and all participants had normal or corrected-to-normal vision. Recruitment of participants was either through an advert on social media, or from the university where the experiment took place, where students received course credits for their participation. Ethical approval was granted from the Ethics Committee of the Department of Psychology prior to data collection. Participants were asked to rate complexity and aesthetic of website screenshots while fixation count and dwelling time were recorded with an eye-tracker.

\subsection{Materials}

Stimuli consisted of screenshot images of university website homepages $(\mathrm{N}=40)$. The 40 images used were selected via a pilot study. In the pilot study, 64 homepage screenshots were first randomly selected from a total of 160 images. Participants to the pilot study $(\mathrm{M}=20.93$ years old, $\mathrm{SD}=2.94$ years), of which half were female, rated the images on one-dimensional scales. The mean aesthetic rating of each image across participants was computed and the top 20 aesthetically-rated and bottom 20 aesthetically-rated images were taken. Image resolution of screenshots was 1366 x 768 pixels. The sample of images for the present experiment thus consisted of 40 pre-calibrated images: Fig. 1 shows two examples of the screenshots used. 

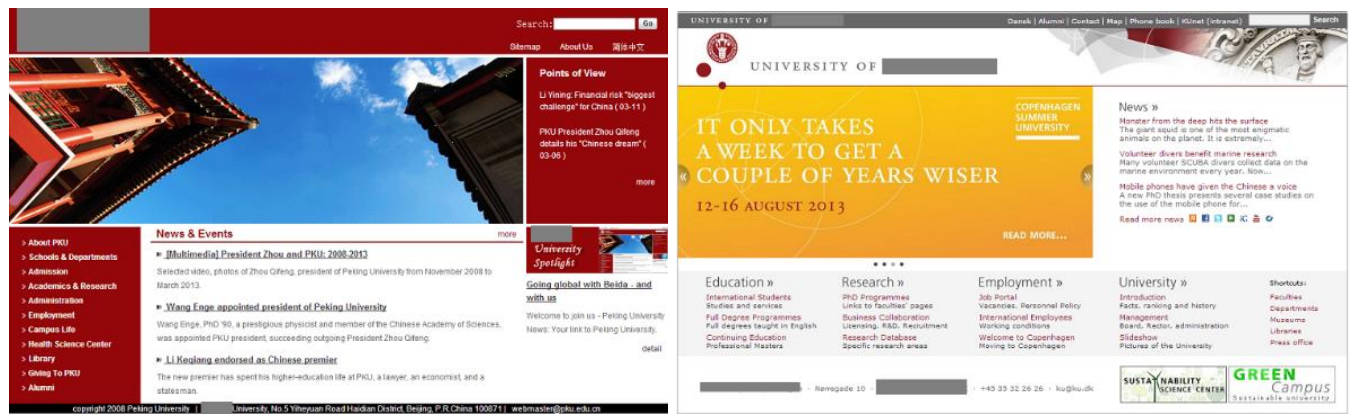

Fig 1. Examples of website images. Note: The names of the universities have been grayed for publications and thus preserve anonymity but were apparent during the study.

An 'Eyelink 1000' eye-tracker, manufactured by SR Research, recorded eye movements sampling data at $1000 \mathrm{~Hz}$ (see Fig.2). Participants used the adjustable SR Research Head Support, positioned $56 \mathrm{~cm}$ away from the monitor. For the experiment a 19" Dell Triton CRT monitor, $36.5 \mathrm{~cm}$ x $28 \mathrm{~cm}$, presented stimuli at $85 \mathrm{~Hz}$.

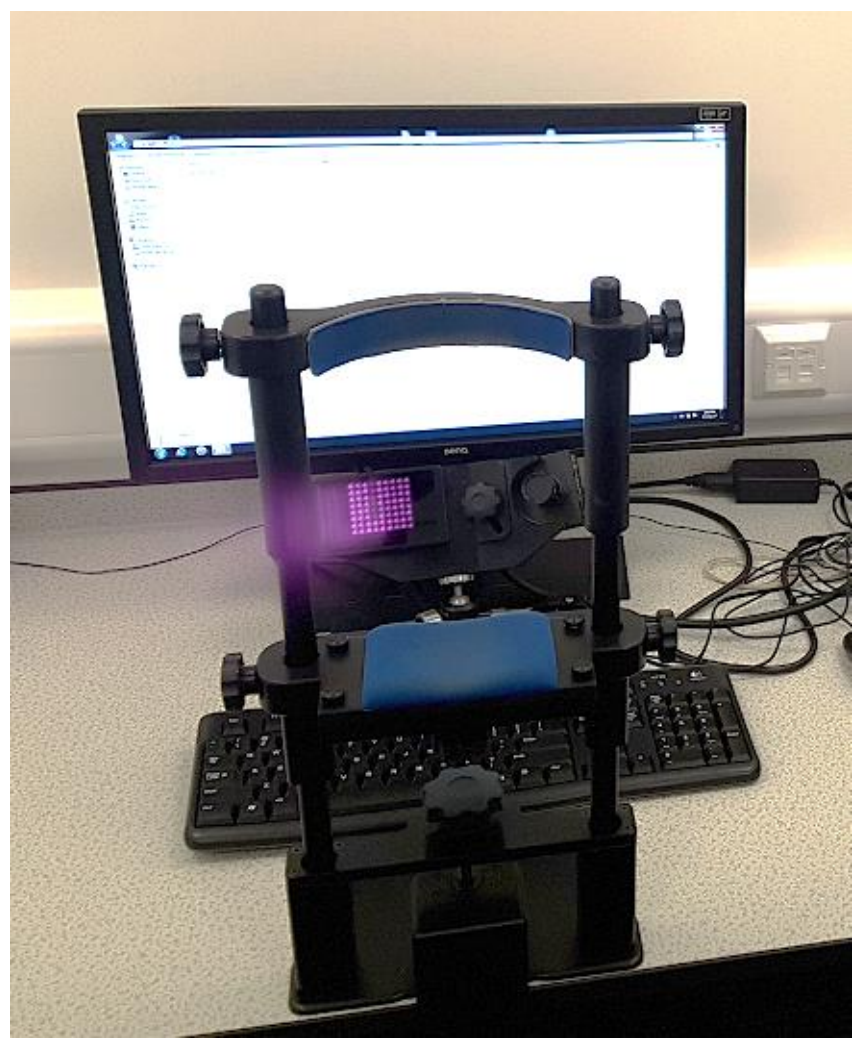

Fig. 2. A photograph of our material: Eyelink 1000.

Note: The monitor has been changed since the experiment took place; the image displays the present configuration. 
We used Likert scales to collect subjective ratings of visual complexity and aesthetic pleasure. One Likert scale was used for collecting ratings of perceptual complexity; anchored at 1 (very easy) to 9 (overload), and another scale was used for collecting ratings of aesthetic pleasure, which was anchored at 1 (extreme negative) and 9 (extreme positive).

\subsection{Procedure}

The participants were informed that they would be presented with 40 university homepage images, and would be required to judge the complexity and aesthetics of each image on a rating scale. The participant's chin and forehead were then situated comfortably in the SR Research head support, which was adjusted accordingly. The left eye of the participant was centred within the viewing field of the eye-tracker camera and was calibrated and validated using a default 9-point grid. Once the left eye had been tracked, participants were instructed to press the ' $O$ ' key on the keyboard, which led to the exposure of an instruction screen explaining the concepts of aesthetic pleasure and perceived complexity. Once participants had read the instructions, they were required to press any key to begin the experimental trials. Each trial commenced with a fixation point in the centre of the display screen. A website image was exposed for 12 seconds. Following exposure, participants were required to rate the image on a Likert scale of Aesthetic Pleasure and Visual Complexity. The Likert rating scales were presented sequentially, until a number was input, completing the trial. The rating phase was not time-restricted and the sequence of trials was randomized. The experiment was conducted in an artificially-lit room and consisted of 40 trials.

\subsection{Measures}

Participants were thus submitted in total to 40 different website screenshots. For each participant and each image, we took four measures:

- Two measures of participants' subjective evaluations of

- Visual complexity, on a 9-point Likert scale. This measure is depending upon the participant's individual perception of each stimuli. It is thus a measure of perceived complexity.

- Aesthetic pleasure, on a 9-point Likert scale.

- Two objective measures of visual complexity:

- The number of eye fixations per image defined as the dwelling of the eye on a location. This is an objective, quantifiable measure of the perceptual effort that the participant has to deploy when analyzing an image.

- The dwelling time on the image as the total time spent by the participant in looking at the image. This measure was to control that the amount of time spent per image was similar across 40 images and participants allowing comparisons of average fixation counts.

\section{RESULTS}

The level of aesthetic pleasure and complexity elicited by a stimulus can be interpreted in relation to the mean of the sample across images and participants. The means indicated that the rating for 
aesthetics was $\mathrm{M}=5.26(\mathrm{SD}=2.09)$, with the mean of visual complexity at $\mathrm{M}=5.00(\mathrm{SD}=2.06)$. Average fixation count across images and participants was 51.18 fixations ( $\mathrm{SD}=11.43$ fixations). Mean values for fixation count, complexity, and aesthetic pleasure were then computed for each image across participants (see Figure 3). These data provide an idea of the variance across images for all recorded measures. Mean dwell time $\mathrm{M}=11.907 \mathrm{~s}(\mathrm{SD}=0.46 \mathrm{~s})$, fixation count $\mathrm{M}=51.18$ $(\mathrm{SD}=1.85)$, aesthetics $\mathrm{M}=5.26(\mathrm{SD}=1.10)$, and complexity $\mathrm{M}=5.00(\mathrm{SD}=.90)$. Fixation duration was $\mathrm{M}=232.90 \mathrm{~ms}(\mathrm{SD}=10.85)$. Figure 3 displays how the three key measures combine for each image.

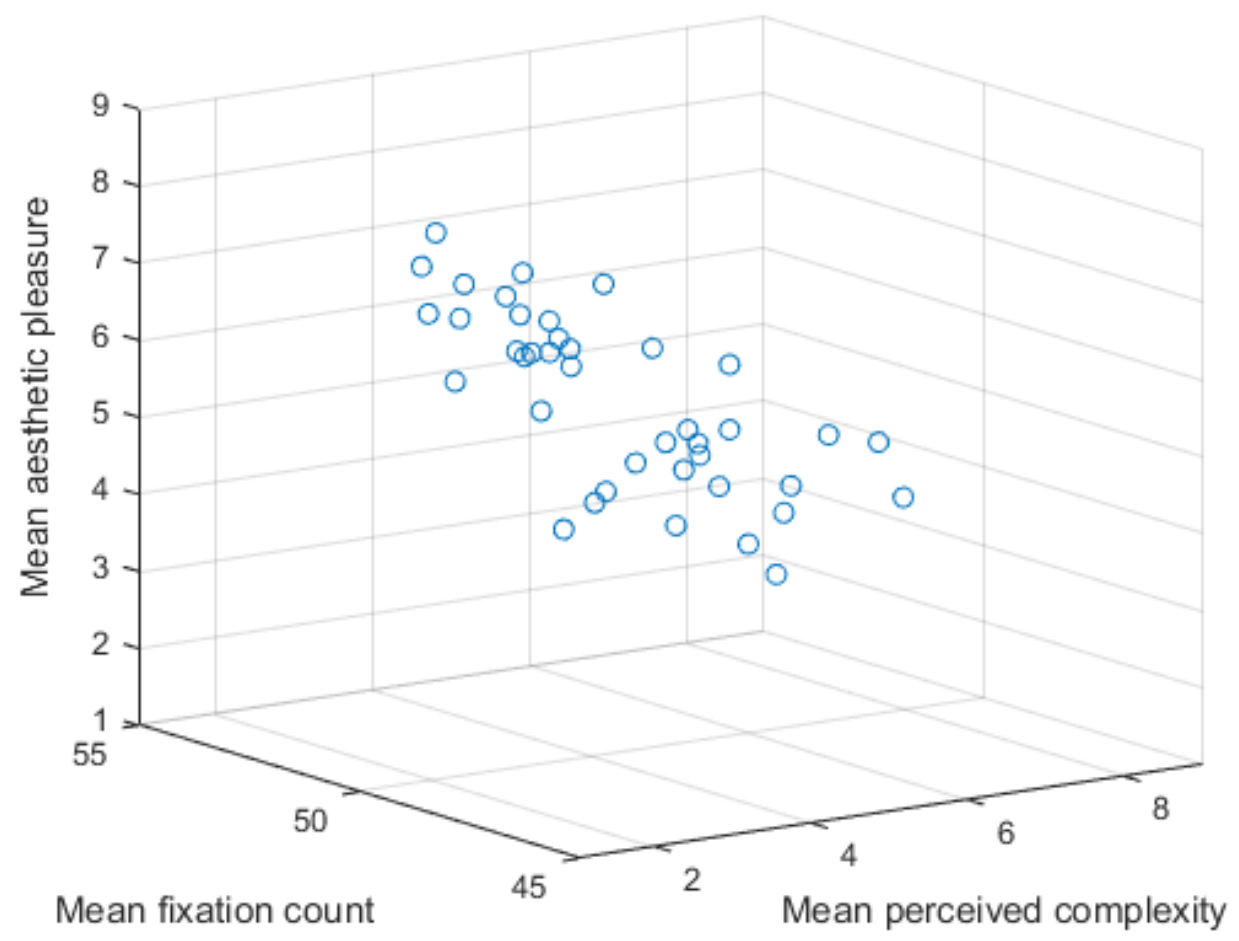

Fig. 3. Three-Dimensional plot of the images. Each point represents an image plotted as a function of its average subjective rating of complexity ( $\mathrm{x}$ axis), its eye fixation count ( $\mathrm{y}$ axis) and its subjective aesthetic pleasure (z axis).

With respect to a relationship between perceived complexity and aesthetics, the two subjective variables correlated negatively with one another, $\mathrm{r}=-.69, \mathrm{p}<.01$. The negative correlation suggests that the more complex a website, the less pleasing it is to the perceiver as indicated by lower subjective ratings of aesthetic pleasure. Linear regression was calculated to investigate the predictive power of visual complexity on aesthetics. A significant regression equation was revealed, $F(1,38)=$ $34.86, \mathrm{p}<.01$, described as aesthetic pleasure $=9.50+$ perceived complexity $*-0.85$. Following the regression model, complexity accounts for $48 \%$ of the variance in aesthetics. Fig. 4 indicates that an increase in the perceived complexity of an image is met with a decrease in its aesthetics. Notably, the figure suggests that the optimum level of perceived complexity is low. Visual complexity also correlated negatively with fixation count, $\mathrm{r}=-.40, \mathrm{p}<.05$. 


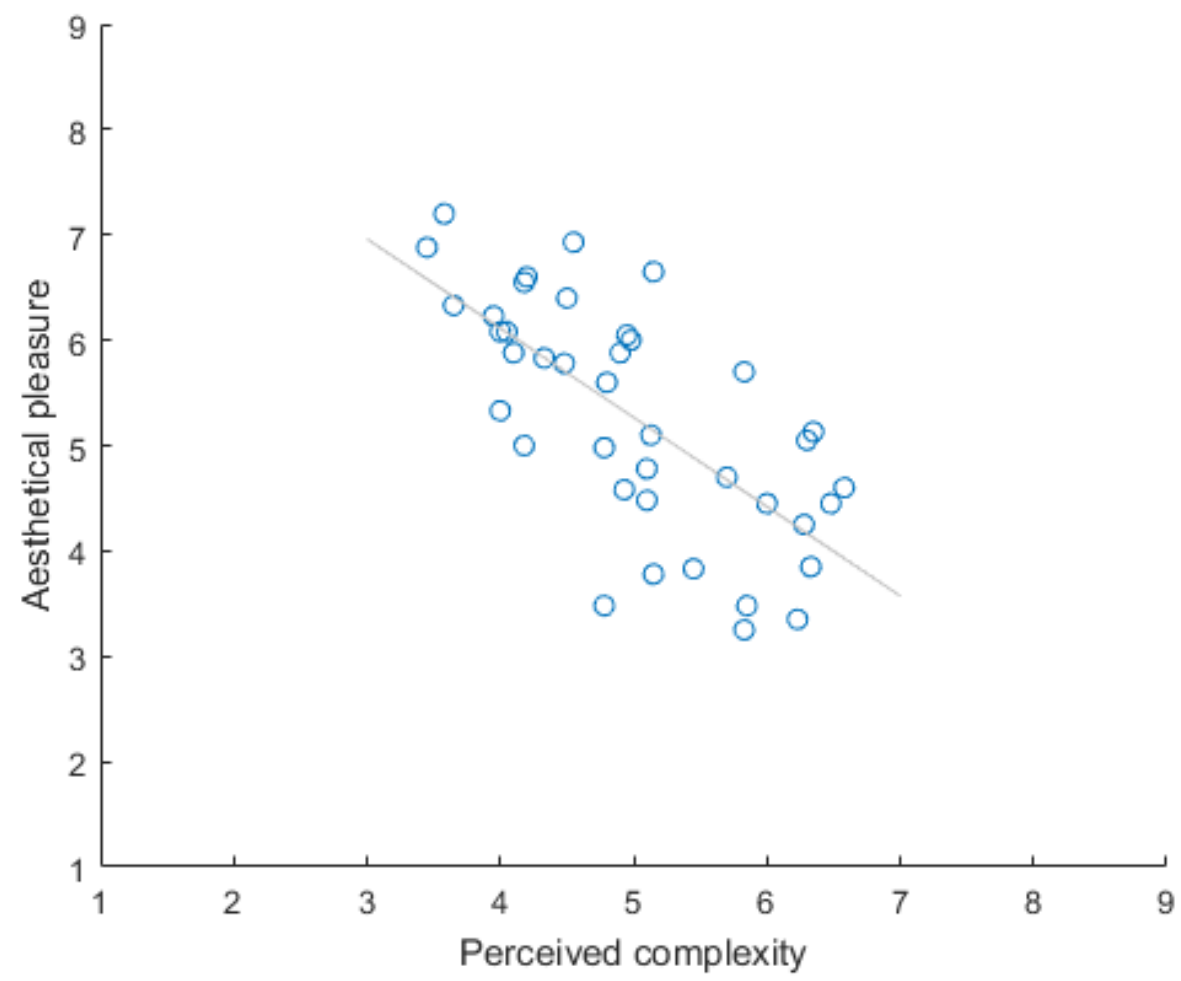

Fig. 4. Linear relationship between Aesthetic pleasure and perceived complexity

A positive relationship was found between fixation count and aesthetic pleasure, $r=.35, p<.05$, indicating that the websites which invite the most fixations are received the most positively (see Fig. 5). Linear regression analysis resulted in a significant positive regression equation, $\mathrm{F}(1,38)=5.21$, $\mathrm{p}<.05$. According to the linear regression, aesthetic pleasure $=-5.30+0.21 *$ fixation count, where fixation count is predictive of $12 \%$ of the variance in aesthetic pleasure. In addition, less eye fixations are met with an increased perception of complexity. Linear regression revealed a significant regression equation, $\mathrm{F}(1,38)=7.11, \mathrm{p}<.05$, described as visual complexity $=14.87-0.19$ * fixation count, where fixation count is predictive of $16 \%$ of the variance in visual complexity. The negative relationship between fixation count and complexity indicates that websites which invite more eye fixations are lower in subjective complexity. 


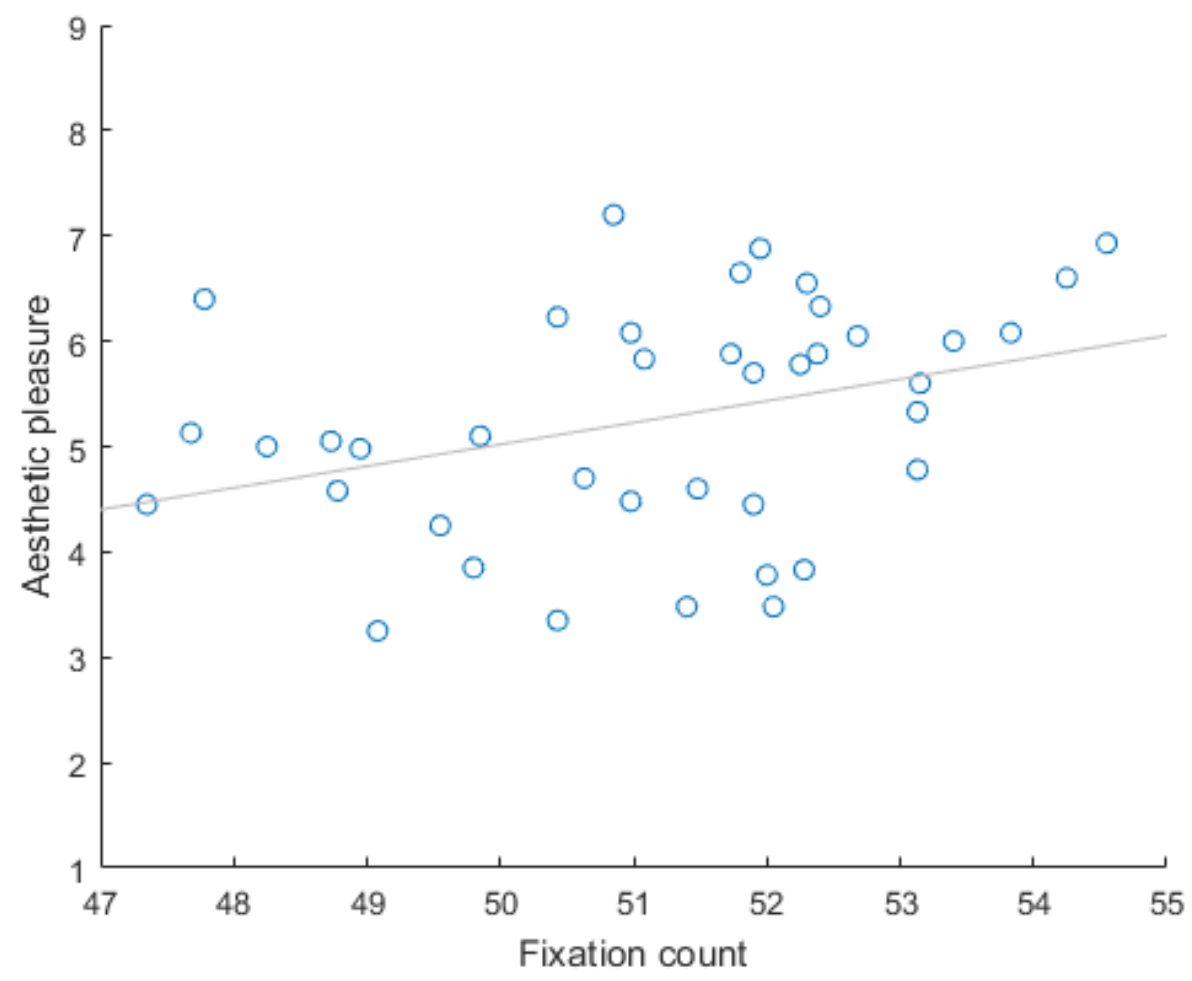

Fig. 5. Linear relationship between aesthetic pleasure and fixation count.

Fig. 5 suggests that fixation count has a positive correlation with aesthetic pleasure. In line with the Figure, websites which are received most negatively invite lower fixations. Conversely, the Figure shows that the most favored websites are scanned more than websites that are less-well received. Dwell time was not found to significantly correlate with complexity, $\mathrm{r}=.26, \mathrm{p}=.10$, or with aesthetics, $\mathrm{r}=-.02, \mathrm{p}=.88$, or fixation count, $\mathrm{r}=.22, \mathrm{p}=.17$. Fixation duration negatively correlated with fixation count, $\mathrm{r}=-.60, \mathrm{p}<.01$ and marginally with aesthetics, $\mathrm{r}=-.28, \mathrm{p}=.07$, but not with complexity $\mathrm{r}=.09, \mathrm{p}=.55$.

\section{DISCUSSION AND CONCLUSIONS}

The purpose of the present study was to investigate the triangulation between objective measures of complexity, and subjective measures of complexity and aesthetics. Using websites, we sought to shed further light on the nature of this relationship, which has yielded conflicting findings in previous studies. Specifically, we predicted that high levels of perceived complexity would result in a low aesthetic rating, which was supported. The prediction that fixation count would correlate positively with visual complexity was unfounded, as fixation count bore a negative linear relation to visual complexity. Finally, fixation count unexpectedly displayed a positive correlation with perceived aesthetics, where a high fixation number was met with a high aesthetic rating.

The results of the present study suggest that websites perceived as low in complexity elicit higher levels of aesthetic appreciation than those that are more visually complex, supporting a growing amount of research that documents a negative relationship [25, 28, 39, 40]. A key feature of working 
memory may help to elucidate the impact of complexity on felt aesthetics. In line with the most prominent model [1], working memory constitutes the way in which we interact with our environment, interfacing our consciousness to information stored in long-term memory, and in doing so, enables us to calculate situations, manipulate information, and predict the consequences of our actions. However, for all its provisions, the capacity of working memory is limited [1, 26]. Anything which exceeds capacity is negatively felt by the perceiver as overwhelming, requiring more cognitive effort to process. Conversely, simple stimuli - expending less processing resources - do not drastically impinge on memory, thus freeing resources for other cognitive functions, which is preferable. In line with the known limitations of memory, objects high in prototypicality, being processed fluently [45], are favorable to the perceiver. The prototypicality of an object refers to the degree of correspondence between the object in its current form and that of its mental concept [30]. The concept is constructed in memory through exposure to a type of object, and future instances of the object are assessed against the prototype in terms of similarities or discrepancies. Studies have indeed documented the role of memory in aesthetic judgements of objects, as research demonstrates that the prototypicality of stimuli influences perceived aesthetic pleasure, where objects of high prototypicality are typically favored $[16,19]$. Human preference for prototypical stimuli extends far beyond evolutionary explanations [37] as, argued by Winkielman, Halberstadt, Fazenderio, and Catty [45], in addition to faces, high preference is consistently reported for non-adaptive objects such as art [19] and musical instruments [32]. As an example, we can consider the two photographs in Fig. 6, taken from a previous study [9] that illustrate the notion of prototypicality. The car on the left, less typical of car fronts than the car on the right, received lower aesthetic ratings. The impact of both prototypicality and complexity on aesthetics has been empirically demonstrated in website perception [40], through findings that websites high in prototypicality and low in visual complexity are more preferable than other combinations of the two variables. In light of these and the above reviewed evidence, we suggest that both perceived visual complexity and aesthetic pleasure elicited from stimuli are consequences of the demands made on working memory for processing a stimulus. Demand can be lessened through the facilitation of prior knowledge about the object, which is preferable to the perceiver and reduces the perception of stimulus complexity.

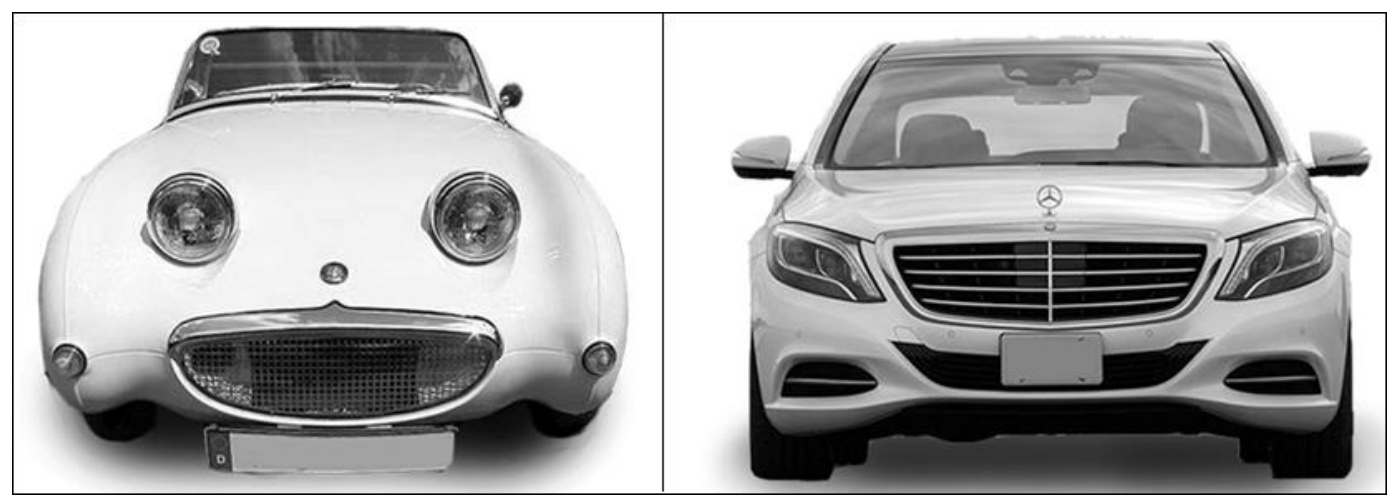

Fig. 6. Two photographs of cars that vary in prototypicality and received different aesthetic ratings [9].

A novel finding in the present study is that the average number of fixation count correlates positively with aesthetic pleasure and negatively with complexity. The fact that all participants observed the 
stimuli for the same amount of time rules out the possibility that the variation in fixations can be accounted for by variance in observation time. Our finding thus strengthens the idea that complexity affects the number of fixations. Regarding the relationship between fixation count and complexity, the finding appears at odds with previous research [9, 42]; most saliently with results obtained using websites [42]. However, manipulation of complexity factors in Wang et al.'s [42] stimuli were mostly visual, whereas the websites used in the present study were university homepages containing both pictures and blocks of text. Verbal information calls for semantic processing, requiring more information to be processed at each eye fixation. Thus presently, a decrease in fixation count was likely due to the additional demands of text comprehension per fixation point, which resulted in increased perceived complexity. Indeed, large amounts of text on a website can appear daunting to users [23]. The notion that more complex stimuli requiring deeper semantic analysis per fixation are less favorable than those that are quickly scanned with many fixations is reinforced by the additional finding of a positive relationship between fixation count and aesthetics. It is important to note that our findings reveal a cautionary aspect of fixation count as a measure of complexity; while fixation count may be used as an objective measure of subjective complexity, the nature of the relationship appears to change as a function of the type of stimuli that are used. Specifically, although fixation count has been shown to positively increase as a function of complexity with visuospatial stimuli $[9,42]$, when text comprehension is required, the converse appears to be true. Together, the present results of fixation count suggest that websites which require few semantically-rich eye fixations are both deemed more complex and felt most unpleasantly by users.

Three limits of the present study should be noted. One limitation is that the study presented a static stimulus as a website. Convinced that dynamical factors play a role in making a website aesthetically pleasing, we limit the scope of the present paper to the first few seconds of contact with the website. It is well established that the first few seconds are crucial, but likely other parameters modulate the aesthetic pleasure derived from the website. The second limit is that the present study did not manipulate levels of prototypicality. Measuring prototypicality would require averaging visual and textual features of many websites, checking against users' experience prior to running an experiment. We are convinced that further studies should be conducted to ascertain the exact influence of knowledge on aesthetic pleasure in website perception. In relation to the present study, future research could investigate the impact of prototypicality on website perception further, with respect to type of processing, to determine whether prototypicality is the strongest predictor of pleasure, or whether it is modulated by type of processing, or vice-versa. The third limit relates to the regression equations. It is difficult to actually put a meaning in the figures, particularly the intercept, as they depend upon the materials used. If the direct interpretation is difficult, the equation still reveals that the variables of perceived complexity and aesthetic pleasure are linearly connected to one another. It is the role of the psychologist now to establish the psychological mechanisms that underpin this linear relationship. Our exploratory study paved the way for future research in perceived complexity. It would be beneficial to replicate our findings with different methodologies so as to specify further the relationship between complexity and aesthetic pleasure.

Our findings have direct implications on the design of websites. Primarily, we have shown that the relationship between complexity and aesthetics stemming from the perception of university websites is negative. In psychology, it has long been demonstrated that chunking information reduces the burden on capacity-limited working memory [26], thus reducing complexity. To make applied use of the acquired knowledge, designers should aim to capitalize on the known limits of the human cognitive architecture [21], in order to create websites that are favorably received. As an example, in accordance with cognitive limits, sites could reduce the amount of text displayed on web pages, making use of key words and bullet points to avoid overloading and discouraging users. Indeed, research suggests that illustrations are much more conducive to positive first impressions than text [31]. Since, though, text is often necessary to explain a website's services, products, or 
purpose, textual information could be grouped into chunks, whilst ensuring consistent font style across segments to avoid complexity. Finally, our findings show that complexity is a multidimensional perceptual phenomenon, further highlighting the caution in measurements of general website complexity [24]. By deepening our knowledge on the dimensions of complexity and their effects on aesthetics, web-design can strive to maximize the positive interaction between webpage and user.

\section{ACKNOWLEDGMENTS}

We would like to thank Martin Guest for his help with setting the eye tracker.

\section{REFERENCES}

[1] Baddeley, A., The episodic buffer: a new component of working memory? Trends in Cognitive Sciences, 2000. 4(11): 417-423.

[2] Belanche, D., L.V. Casaló, and M. Guinalíu, Website usability, consumer satisfaction and the intention to use a website: The moderating effect of perceived risk. Journal of Retailing and Consumer Services, 2012. 19(1): 124132 .

[3] Berlyne, D.E., Aesthetics and Psychobiology. 1971, New York: Century-Crofts.

[4] Berlyne, D.E., Studies in the New Experimental Aesthetics - steps toward an Objective Psychology of Aesthetic Appreciation. 1974, Washington: Hemisphere Publishing.

[5] Buettner, R., The relationship between visual website complexity and a user's mental workload: A NeuroIS perspective, in Information Systems and Neuroscience. 2017, Springer. p. 107-113.

[6] Butkiewicz, M., H.V. Madhyastha, and V. Sekar. Understanding website complexity: measurements, metrics, and implications. in Proceedings of the 2011 ACM SIGCOMM conference on Internet measurement conference. 2011: ACM.

[7] Chase, W.G. and H.A. Simon, Perception in chess. Cognitive Psychology, 1973. 4(1): 55-81.

[8] Chassy, P. and F. Gobet, Visual search in ecological and non-ecological displays: Evidence for a non-monotonic effect of complexity on performance. PLoS ONE, 2013. 8(1): e53420.

[9] Chassy, P., T.A. Lindell, J.A. Jones, and G.V. Paramei, A relationship between visual complexity and aesthetic appraisal of car front images: an eye-tracker study. Perception, 2015. 44(8-9): 1085-1097.

[10] Cheng, H.-I. and P.E. Patterson, Iconic hyperlinks on e-commerce websites. Applied Ergonomics, 2007. 38(1): 6569.

[11] Cober, R.T., D.J. Brown, P.E. Levy, A.B. Cober, and L.M. Keeping, Organizational web sites: Web site content and style as determinants of organizational attraction. International Journal of Selection and Assessment, 2003. 11(2-3): 158-169.

[12] Deng, L. and M.S. Poole, Affect in web interfaces: a study of the impacts of web page visual complexity and order. Mis Quarterly, 2010: 711-730.

[13] Geissler, G.L., G.M. Zinkhan, and R.T. Watson, Web home page complexity and communication effectiveness. Journal of Association for Information Systems, 2001. 2(2): 1-46.

[14] Geissler, G.L., G.M. Zinkhan, and R.T. Watson, The influence of home page complexity on consumer attention, attitudes, and purchase intent. Journal of Advertising, 2006. 35(2): 69-80.

[15] Guo, Y.M. and D. Hall. Website complexity: Objective versus subjective measures. in MWAIS 2009 Proceedings. 2009.

[16] Halberstadt, J.B. and G. Rhodes, It's not just average faces that are attractive: Computer-manipulated averageness makes birds, fish, and automobiles attractive. Psychonomic Bulletin \& Review, 2003. 10: 149-156.

[17] Hargreaves, D.J., The effects of repetition on liking for music. Journal of Research in Music Education, 1984. 32: 35-47.

[18] Heath, T., S.G. Smith, and B. Lim, Tall buildings and the urban skyline: The effect of visual complexity on preferences. Environment and Behavior, 2000. 32(4): 541-556.

[19] Hekkert, P. and P.C.W. Wieringen, Complexity and prototypicality as determinants of the appraisal of cubist paintings. British Journal of Psychology, 1990. 4: 483-513.

[20] Huang, Z. and M. Benyoucef, Usability and credibility of e-government websites. Government Information Quarterly, 2014. 34(1): 584-595. 


\section{Chassy, Fitzpatrick, Jones and Pennington Journal of Interaction Science (2017) 5:3}

[21] Jones, J., J. Fitzpatrick, and P. Chassy, The cognitive engineering of memory in educational website design. Advances in Social Sciences Research Journal, 2015. 2(5): 115-121.

[22] Krauss, K. The value and process of visual aesthetics as communication tool for web pages. in International Business Informatics Challenge. 2006.

[23] Krug, S., Don't Make Me Think! A Common Sense Approach to Web Usability. 2006, Berkeley, California: New Riders.

[24] Mai, R., Hoffmann, U. Schwarz, T. Niemand, and J. Seidel, The shifting range of optimal web site complexity. Journal of Interactive Marketing, 2014. 28(2): 101-116.

[25] Michailidou, E., S. Harper, and S. Bechhofer, Visual complexity and aesthetic perception of Web pages, in 26th annual ACM international conference on Design of communication. 2008: New York: USA.

[26] Miller, G.A., The magical number seven. Plus or minus two: some limits on our capacity for processing information. Psychological Review, 1956. 62(2): 81-97.

[27] Nadkarni, S. and R. Gupta, A task-based model of perceived website complexity. Mis Quarterly, 2007: 501-524

[28] Pandir, M. and J. Knight, Homepage aesthetics: the search for preference factors and the challenges of subjectivity. Interacting with Computers in Human Behavior, 2006. 18: 1351-1370.

[29] Poole, A. and L. Ball, Eye tracking in HCI and usability research, in Encyclopedia of Human Computer Interaction, C. Ghaoui, Editor. 2006, Idea Group Inc.: Calgary, Canada. p. 211-223.

[30] Rosch, E.H., Natural categories. Cognitive Psychology, 1973. 4: 328-350.

[31] Schenkman, B.N. and F.U. Jönsson, Aesthetics and preferences of web pages. Behaviour \& Information Technology, 2000. 19(5): 367-377.

[32] Sen, D. and G. Lindgaard, The impact of prototypicality on aesthetic evaluation: towards a better understanding of affective components of interface design, in IADIS International Conference on Interfaces and Human Computer Interaction. 2008: Amsterdam, Netherlands.

[33] Simon, H.A., The architecture of complexity, in Facets of systems science. 1991, Springer. p. 457-476.

[34] Sporns, O., Network analysis, complexity, and brain function. Complexity, 2002. 8(1): 56-60.

[35] Stevenson, J.S., G.C. Bruner, and A. Kumar, Webpage background and viewer attitudes. Journal of Advertising Research, 2000. 40: 29-34.

[36] Stickel, C., M. Ebner, and A. Holzinger. The XAOS Metric - Understanding visual complexity as measure of usability. in 6th Symposium of the Workgroup Human-Computer Interaction and Usability Engineering. 2010. Klagenfurt, Austria.

[37] Symons, D., Evolution of human sexuality. 1979, New York: Oxford University Press.

[38] Thielsch, M.T., I. Blotenberg, and R. Jaron, User evaluation of websites: From first impression to recommendation. Interacting with Computers, 2013. 26(1): 89-102.

[39] Tuch, A.N., J.A. Bargas-Avilaa, K. Opwis, and F.H. Wilhelm, Visual complexity of websites: Effects on users' experience, physiology, performance, and memory. International Journal of Human-Computer Studies, 2009. 67: 703-715.

[40] Tuch, A.N., E.E. Presslaber, M. Stöcklin, K. Opwis, and J.A. Bargas-Avila, The role of visual complexity and prototypicality regarding first impression of websites: Working towards understanding aesthetic judgments. International Journal of Human-Computer Studies, 2012. 70(11): 794-811.

[41] Wang, H.-F., Picture perfect: Girls' and boys' preferences towards visual complexity in children's websites. Computers in Human Behavior, 2014. 31: 551-557.

[42] Wang, Q., Yang, M. Liu, Z. Cao, Q. Ma,, An eye-tracking study of website complexity from cognitive load perspective. Decision Support Systems, 2014. 62: 1-10.

[43] Wang, Y.J., M.D. Hernandez, and M.S. Minor, Web aesthetics effects on perceived online service quality and satisfaction in an e-tail environment: The moderating role of purchase task. Journal of Business Research, 2010. 63: 935-942.

[44] Wills, R.F., Organizational recruitment web sites: The influence of web site aesthetics on initial affective reactions to the site and subsequent attraction to the organization. 2007, Clemson University.

[45] Winkielman, P., Halberstadt, T. Fazenderio, and S. Catty, Prototypes are attractive because they are easy on the mind. Psychological Science, 2006. 17: 799-806.

[46] Xiang, X., J. Chen, and Y. Ye. Using websites to attract applicants: a signal-based model of online organizational attraction. in Engineering Management Conference, 2005. Proceedings. 2005 IEEE International. 2005: IEEE.

(C) 2017 by the authors. This article is an open access article distributed under the terms and conditions of the Creative Commons Attribution License (http://creativecommons.org/licenses/by/4.0/). 
Chassy, Fitzpatrick, Jones and Pennington Journal of Interaction Science (2017) 5:3

APPENDIX A - Mean values for the 40 websites

Table 1. Descriptive statistics for the 40 stimuli.

\begin{tabular}{lllllll} 
Picture & Count & & Nice & \multicolumn{2}{l}{ Complex } \\
& M & SD & M & SD & M & SD \\
1 & 47.35 & 11.28 & 4.45 & 1.57 & 6.48 & 1.81 \\
2 & 52.05 & 12.39 & 3.48 & 2.15 & 4.78 & 2.28 \\
3 & 52.28 & 10.87 & 3.82 & 2.06 & 5.45 & 2.08 \\
4 & 51.40 & 9.96 & 3.48 & 1.85 & 5.85 & 2.03 \\
5 & 48.78 & 9.47 & 4.58 & 1.85 & 4.93 & 1.95 \\
6 & 47.68 & 12.72 & 5.13 & 1.64 & 6.35 & 2.07 \\
7 & 49.08 & 10.41 & 3.25 & 1.85 & 5.82 & 2.17 \\
8 & 50.42 & 10.27 & 3.35 & 1.96 & 6.23 & 2.37 \\
9 & 50.98 & 12.12 & 4.48 & 1.63 & 5.10 & 1.52 \\
10 & 49.55 & 12.07 & 4.25 & 1.66 & 6.28 & 1.74 \\
11 & 48.73 & 9.33 & 5.05 & 1.97 & 6.30 & 1.57 \\
12 & 52.00 & 14.21 & 3.78 & 2.01 & 5.15 & 1.97 \\
13 & 53.13 & 12.60 & 4.77 & 1.53 & 5.10 & 1.87 \\
14 & 51.48 & 10.08 & 4.60 & 1.63 & 6.58 & 1.74 \\
15 & 48.25 & 10.31 & 5.00 & 2.16 & 4.17 & 1.85 \\
16 & 49.85 & 11.25 & 5.10 & 1.96 & 5.13 & 2.05 \\
17 & 51.90 & 9.59 & 4.45 & 2.01 & 6.00 & 2.08 \\
18 & 48.95 & 10.65 & 4.98 & 1.69 & 4.78 & 1.72 \\
19 & 49.80 & 10.76 & 3.85 & 2.08 & 6.32 & 2.08 \\
20 & 50.63 & 12.01 & 4.70 & 1.80 & 5.70 & 2.00 \\
21 & 53.82 & 11.53 & 6.07 & 1.79 & 4.05 & 1.87 \\
22 & 52.38 & 13.06 & 5.88 & 1.90 & 4.90 & 1.71 \\
23 & 50.97 & 12.40 & 6.07 & 1.54 & 4.00 & 1.66 \\
24 & 52.30 & 13.05 & 6.55 & 1.57 & 4.18 & 1.77 \\
25 & 51.95 & 11.25 & 6.88 & 1.81 & 3.45 & 2.00 \\
26 & 54.55 & 9.61 & 6.92 & 1.94 & 4.55 & 2.01 \\
27 & 53.13 & 9.34 & 5.33 & 2.12 & 4.00 & 1.54 \\
28 & 52.40 & 13.47 & 6.32 & 1.72 & 3.65 & 1.72 \\
29 & 51.72 & 12.35 & 5.88 & 1.81 & 4.10 & 1.85 \\
30 & 47.78 & 10.34 & 6.40 & 1.77 & 4.50 & 1.43 \\
31 & 53.15 & 8.96 & 5.60 & 1.37 & 4.80 & 1.80 \\
32 & 51.90 & 9.60 & 5.70 & 1.62 & 5.83 & 1.57 \\
33 & 51.07 & 9.16 & 5.83 & 1.92 & 4.33 & 1.77 \\
34 & 50.85 & 11.57 & 7.20 & 1.94 & 3.58 & 2.28 \\
35 & 12.80 & 10.14 & 6.65 & 1.61 & 5.15 & 1.88 \\
36 & 12.68 & 5.78 & 1.86 & 4.48 & 1.59 \\
37 & 14.16 & 6.60 & 1.77 & 4.20 & 1.98 \\
38 & 53.45 & 6.05 & 1.74 & 4.95 & 1.88 \\
39 & 5.00 & 1.62 & 4.98 & 1.76 \\
40 & 5.98 & 6.23 & 1.61 & 3.95 & 2.05
\end{tabular}

